\title{
FOR PEER REVIEW
}

\section{Measurement Bias, Sex Differences, and the Implications of Substantial Theories}

\author{
Jordan Lasker ${ }^{\mathrm{a}}$ \\ aTexas Tech University, Lubbock TX, USA. Email: jlasker@ttu.edu
}

\begin{abstract}
ARTICLE HISTORY
Compiled December 9, 2021

ABSTRACT

Personality researchers frequently invoke theories to explain why groups differ in terms of measured personality traits. Their explanations often come with additional predictions about the models used to measure those traits. Particularly, they often suggest that the models will have the same parameters for members of different groups or, inversely, that the parameters will differ so much that they explain why groups differ. I argue that theoretical predictions that are easy to test but are not often thought about can help provide support for or threaten the viability of certain theoretical explanations. In the current study, I used the case of the so-called Gender Equality Paradox and in a sample of 59 countries, showed that the data are not consistent with a wide range of existing explanations.
\end{abstract}

\section{KEYWORDS}

Measurement Invariance; Big Five; Personality; Group Differences; Gender

Equality Paradox; Confirmatory Factor Analysis

\section{Introduction}

Sex differences in personality are widely discussed and have been the topic of a great deal of research. The size of the personality differences between men and women is heavily contested, with proponents of small differences (Hyde, 2005; Zell et al., 2015) coexisting in clear contrast with proponents of large ones (Booth \& Irwing, 2011; Del Giudice et al., 2012; Kaiser et al., 2020). The different methods used by advocates of either position have sparked some controversy (e.g., Hyde, 2012; Del Giudice, 2013), and debate in this area at all levels is by no means settled.

\subsection{Substantial Explanations and the Assumption of Substance}

Beyond mere characterization of the differences between men and women, there is a large literature that purports to explain these differences. Explanations frequently invoke notions such as "gender equality" (Costa et al., 2001; Schmitt, 2015; Giolla \& Kajonius, 2018; Fors Connolly et al., 2020)1 , "economic development" (Schmitt et al., 2009), "culture" (Schmitt et al., 2017), "development" (Bolle et al., 2015), "evolution"

\footnotetext{
${ }^{1}$ The relationship between gender equality and sex differences in personality and several other traits has been dubbed the Gender Equality Paradox because of the unintuitive finding that sex differences tend to be larger in more gender-equal countries.
} 
(Benenson et al., 2021), "ecological stress" (Kaiser, 2019), "genetics" (Ngun et al., 2011; Richardson \& Boutwell, 2020), "neurobiology" (Stam et al., 2019; van Eijk et al., 2021), and so on ${ }^{2}$. The common current through all the explanations and most others out there is that they are substantial; this large literature tacitly holds that the differences between the sexes observed in its' studies are real and caused by some thing or set of things that affects changes in people's psychology. I will call this an assumption of substance.

The assumption of substance has been tested in a variety of ways. Broadly speaking, the two most common ways this assumption is tested are multigroup confirmatory factor analysis (MGCFA) and the assessment of differential item functioning (DIF). These methods both involve testing for differences in the parameters of models in which personality is depicted as a latent common cause for the correlations between items from a measurement instrument. Differences in model parameters (i.e., "bias") like intercepts and regression slopes signify differences in how members of different groups understand the instrument (see, e.g., van de Schoot et al., 2012, p. 490; Fokkema et al., 2013, p. 521). Departures from parameter equality between groups can affect the mean scores groups obtain, potentially explaining the entirety of why groups differ, or even making differences smaller than they ought to be in the absence of bias. When parameter equality - otherwise known as measurement invariance - is observed, it indicates that different groups interpret the instrument the same way and that the differences in means and variances for a well-fitting model can be attributed to real differences in the construct the questionnaire measures if it, indeed, measures a coherent construct at all.

Papers that have tested for measurement invariance for personality by sex include Orri et al.'s (2018) investigation of the Affective Neuroscience Personality Scales, Suzuki et al.'s (2019) look into the DSM-5 Pathological Personality Trait test, or Marsh et al.'s (2010) exploration of the NEO-FFI, among many others (see Dong \& Dumas, 2020). Per Dong \& Dumas, the general tenor of these results is such that the "configural model invariance of all these studies [on gender] was supported, the majority of them displayed metric invariance, and 13 [of 29] studies further show[ed] that their personality measurement tools [could] hold full scale invariance or a higher level" (p. 6). When it comes to sex, measurement invariance was, thus, often supported. Despite this, there is a major problem with how measurement invariance is often interpreted, a problem of interpreting its magnitude. Millsap (2005) described four problems in testing measurement invariance; one of these was the effect size issue, whereby when we find evidence that measurement invariance is violated, the analysis ends there without any information regarding how large the effect of test bias is. In fact, a given model might showcase some evidence for bias in the sense that parameters differ significantly at some threshold, but the actual effect on means and variances might be negligible. Dong \& Dumas had this to say: "[V]ery few studies [in their systematic review] interpreted measurement invariance by referencing effect sizes. Effect sizes in testing [measurement invariance] could indicate whether the detected DIF or non-invariance is substantial, which means they should be incorporated with other evidence to determine if the personality measures are valid for comparing group differences. In many cases, a small number of DIF items have negligible effects on comparisons of personality scores, so effect sizes can help indicate whether the detection of DIF items is a substantial issue" (p. 7; see also pp. 9-10).

${ }^{2}$ The role of sex hormones is frequently brought up in non-academic discussions of sex differences but evidence for a contribution to sex differences in direct investigations has been, so far, poor, as in Udry \& Talbert (1988). 


\subsection{The Theoretical Suggestion of Measurement Invariance Violation}

In addition to the practical benefits of measuring how large the effect of bias is, computing the effects and signs of measurement bias can be useful for substantial theory testing because many theories suggest or imply the presence of measurement bias. For example, take Guimond et al. (2007); these researchers supposed that the perplexing relationship between sex differences and country-level gender equality whereby more gender-equal countries yielded larger sex differences could be explained by an effect of social comparison where individuals in less gender-equal countries tended to compare themselves to their own sex more so than those from gender-equal countries, who tend, instead, to compare themselves to their own and the other sex, thus exaggerating sex differences. Unless this explanation suggests that self-perception drives actual changes in personality, then it implies changes in item parameters in one or another (or both) sex(es) such that bias is greater in more gender-equal countries because the true value for the personality trait under investigation is unchanged but it nonetheless diverges from the observed value. Moreover, if both sexes are affected, then the effect must be homogeneous within- and between-groups to avoid detection in sufficiently large samples. If it is so small or subtle that it needs infeasible sample sizes then in this domain where samples numbering in the hundreds of thousands are relatively common, it must be a very small effect or subject to some sort of phenomenon like range restriction that limits our ability to effectively detect it. Regardless, when an effect is described as homogeneous in large populations where manipulation is infeasible, it enters the realm of pseudoscience; such a hypothetical is unfalsifiable, so because it is unlikely personality is actually changed by social comparison and no one could reasonably expect there to be anything less than substantial heterogeneity in the experience of phenomena like "gender equality" within a country, such an evasion of hard theory testing is dismissed as easily as it is proposed.

Regardless of its considerable practical and theoretical utility, very few of the attempts to assess measurement invariance by sex have included effect sizes. One of the few examples I am aware of (Nye et al. 2016) utilized $d_{D T F}$, a scale-wise measure of the amount of bias in a set of items used to indicate a measure, and $d_{M A C S}$, the same sort of measure but for MGCFAs. They found that the amount of bias indicated by $d_{D T F}$ was small, ranging from $|d|=0$ to 0.16 , whereas the amount of bias indicated by $d_{M A C S}$ ranged from 0.05 to 0.26 . There is a critical difference between these two measures: the former is signed, and the latter is not. Unlike an unsigned measure, a signed measure indicates both the magnitude and the direction of an effect. To understand the aggregate amount of bias - since the same test can include items biased in favor of and against either group - one needs a signed measure, lest they conflate all bias with bias with respect to a particular group. When testing theories, it is likely that a signed effect size would greatly aid interpretation as, without it, the effects would be unable to be reconciled with theoretical predictions, either in magnitude or direction.

\subsection{Measuring Bias in Multigroup Confirmatory Factor Models}

Fortunately for practitioners of MGCFAs, Nye et al. (2019) have recently proposed a signed version of $d_{M A C S}$ called $d_{M A C S_{-} \text {Signed }}$. Formula-wise, $d_{M A C S}$ and $d_{M A C S \_ \text {Signed }}$ are as follows: 


$$
d_{M A C S}=\frac{1}{S D_{j \text { Pooled }}} \sqrt{\int\left(\hat{Y}_{j 1}-\hat{Y}_{j 2} \mid \eta\right)^{2} \cdot f_{2}(\eta) d \eta}
$$

and

$$
d_{\text {MACS_Signed }}=\frac{1}{S D_{j \text { Pooled }}} \int\left(\hat{Y}_{j 1}-\hat{Y}_{j 2} \mid \eta\right) \cdot f_{2}(\eta) d \eta
$$

$d_{\text {MACS_Signed }}$ can be thought of as the unsquared version of $d_{M A C S}$. Because of the loss of squaring, these effect size measures are no longer equivalent, but it is the former of the two that provides the correct magnitude, even though the latter yields the correct direction, and they can be equivalent with invariant loadings. As such, I propose $d_{\text {MACS_True }}{ }^{3}$, which is given as

$$
d_{\text {MACS_True }}=\left\{\begin{array}{l}
d_{\text {MACS }} \text { if } d_{\text {MACS_Signed }} \geq 0 \\
d_{\text {MACS }} \times-1 \text { if } d_{\text {MACS_Signed }}<0
\end{array}\right.
$$

This function provides both the proper magnitudes and directions of effects for bias in MGCFAs involving two groups, when a single factor model is used. Code is provided $^{4}$.

\subsection{Purpose of the Current Study}

As explained above, $d_{\text {MACS_True }}$ and similar effect sizes can be brandished as tools to shine light on the viability of various hypotheses about sex differences in personality. There are abundant examples of papers on international sex differences in personality; providing estimates of the amount of bias in personality measurement between the sexes across many countries can help to assess whether substantial explanations for their observations are truly capable of providing researchers with signal rather than noise. The purpose of the present study is to assess whether there is sex-related bias in the measurement of personality, how large it is, and if there are patterns in its presentation across countries. The resulting information and the methods used to obtain it can be freely leveraged by substantial researchers for their own theory crafting.

\section{Method}

\subsection{Materials}

The International Personality Item Pool (IPIP) is a pool of 3,320 publicly-available and freely-accessible personality items that have been used in the construction of hundreds of personality scales (Goldberg et al., 2006). 120 of these items were included in the IPIP-NEO 120, a 120-item collection of questions distilled from the original 300-item

\footnotetext{
${ }^{3}$ From section 2 onwards, $d_{\text {MACS_True }}$ is referred to as $d_{M A C S}$.

${ }^{4}$ https://rpubs.com/JLLJ/dMACSTrue.
} 
IPIP-NEO and intended to measure thirty facets of the Big Five personality factors with a reasonably short questionnaire (Johnson, 2014). Based on the validity evidence provided in the IPIP-NEO 120's norming, we expect to observe six facets for each of five factors in Anglophone countries and, perhaps, evidence for additional crossloadings or fewer factors in less economically developed countries (Lukaszewski et al., 2017; Lasker et al., 2021).

\subsection{Participants}

Participants were members of Johnson's (2005) internet-based sample of persons who took the IPIP-NEO 120 online. I used the IPIP-NEO 120 sample instead of the IPIPNEO 300 sample because the former was nearly twice as large. The individuals involved in this sample discovered the personality test online independently or via referral by word-of-mouth and were not actively recruited by Johnson. The total sample comprised 619,150 individuals from 241 countries but since the present study was geared towards testing measurement invariance by sex within each country, I subset to countries with a Global Gender Gap Report (Schwab et al., 2015) entry and well-balanced samples of approximately 100 per sex, leaving me with a total sample including 59 countries and 604,561 complete responses. Johnson (2005, 2014) provided additional detail about the sample. The data and the facet and factor scoring directions can be accessed at https://osf.io/wxvth. The factor models are based on the factor scoring directions and the "General Factor of Personality" or GFP was scored as suggested by Musek (2007).

\subsection{Data Analytic Procedures}

\subsubsection{Measurement Invariance and $d_{M A C S}$}

I tested measurement invariance by sex once per factor or general factor for a total of six tests of measurement invariance with respect to sex by country. Single-factor models were utilized because $d_{M A C S}$ is presently incapable of being utilized with respect to multiple factors and to avoid potential biases in the evaluation of model fit due to the presence of many, for each test, superfluous factors that might serve to alter loadings in unforeseen ways. In short, simplicity was sought for analytic simplicity since the sample size being used is very large and the number of models to fit is also. When models were fit, they were only fit to the configural stage of measurement invariance testing (Beaujean, 2014, p. 58) and both the factor loadings and intercepts were considered to be biased even if testing would have shown otherwise ${ }^{5} . d_{M A C S}$ was computed based on these presumed-biased parameters and the $d_{M A C S}$ values for each country were the sum of $d_{M A C S}$ values for the sex comparisons within them.

\subsubsection{Regressions}

I performed a series of robust regressions using HC5 standard errors (Cribari-Neto et al., 2007). These regressions were run without and with $\sqrt{N}$ weights as a robustness

\footnotetext{
${ }^{5}$ This may induce artificial DIF, but as stated by Hagquist \& Andrich (2017) in reference to a comparable model, "Neither in uniform DIF nor in non-uniform DIF, does artificial DIF balance out real DIF with respect to group differences in the person estimates" (p. 3), so if the estimates are off, they are likely at least directionally consistent. This form of bias is also unlikely theoretically, since it is hard to imagine that even within each facet, the questions will suffer from a uniform bias.
} 
Table 1. Mean $d_{M A C S}$ by Factor

\begin{tabular}{lcc}
\hline & & \\
Factor & Unweighted & $\sqrt{N}$ Weighted \\
\hline Openness & 0.075 & 0.038 \\
Conscientiousness & -0.002 & -0.028 \\
Extraversion & 0.007 & -0.010 \\
Agreeableness & 0.022 & -0.007 \\
Neuroticism & 0.017 & 0.044 \\
GFP & 0.023 & 0.033 \\
\hline
\end{tabular}

All results are rounded.

check because some points represented much larger samples than others and might thus be more reliable. Bias was conceptualized as the $d_{M A C S}$ values, which were utilized alongside estimated mean differences in terms of Cohen's $d$ and the 2015 version of a metric known as the Global Gender Gap Index, a World Economic Forum-created index of country-level gender inequality (Schwab et al., 2015). The reason this index was used was because it is readily available and it is very common in the literature to assess the relationship between sex differences in a variety of outcomes and "gender equality", as measured by this and other indices (e.g., Stoet et al., 2016; Breda et al., 2020; S. S. Richardson et al., 2020; Cuevas et al., 2021). As such, I sought to provide evidence about whether bias covaried with gender equality as indexed by this commonly used measure.

\section{Results}

All code and results can be found at https://rpubs.com/JLLJ/GEPJ120. That page also includes negative controls in the form of regressions against sample size. None of those relationships were significant.

\section{1. $d_{M A C S}$ Across Countries}

Table 1 contains the average levels of $d_{M A C S}$ by country. No value exceeded $d=0.1$. To contextualize these results, the value for GFP can be compared to Mahalanobis' $D^{6}$ for the factor sumscores. This value was $D=0.305$ when unweighted (or 0.301 when weighted), which means multivariate differences may have been 13.26 (9.12) times as large as the effect of bias. Though not the topic of this study, mean bias accounted for much less than observed mean sex differences in personality.

\subsection{Regression Results}

\subsubsection{Bias versus Sex Differences}

Table 2 contains results from the regressions of $d_{M A C S}$ against sex differences by country. There were, evidently, no significant relationships between $d_{M A C S}$ and sex differences. This was true regardless of weighting.

\footnotetext{
${ }^{6}$ These values may seem small compared to the rest of the literature. This is because I computed them based on the scores for the factors and not the facets. When additional imperfectly correlated variables are included in the computation of Mahalanobis' $D$, differences can tautologically increase provided there are any differences in the newly introduced variables. When I compute $D$ based on facets, my value of $D$ is comparable to earlier literature, but I computed it this way to maintain comparability to the $d_{M A C S}$ for the GFP.
} 
Table 2. $d_{M A C S}$ versus Sex Differences

\begin{tabular}{lcc}
\hline Factor & Unweighted & $\sqrt{N}$ Weighted \\
\hline Openness & $0.328(0.386)$ & $0.342(0.421)$ \\
Conscientiousness & $-0.194(0.565)$ & $-0.741(0.567)$ \\
Extraversion & $0.161(0.126)$ & $0.107(0.106)$ \\
Agreeableness & $0.228(0.468)$ & $0.154(0.887)$ \\
Neuroticism & $0.149(0.146)$ & $0.142(0.225)$ \\
GFP & $0.029(0.209)$ & $0.0001(0.205)$ \\
\hline Unit is B. SEs are in & parentheses.
\end{tabular}

Table 3. $d_{M A C S}$ versus Gender Equality

\begin{tabular}{|c|c|c|}
\hline Factor & Unweighted & $\sqrt{N}$ Weighted \\
\hline Openness & $-0.247(0.175)$ & $-0.256(0.130)$ \\
\hline Conscientiousness & $0.080(0.149)$ & $0.023(0.123)$ \\
\hline Extraversion & $-0.404(0.078) * * *$ & $-0.350(0.080)^{* * *}$ \\
\hline Agreeableness & $0.305(0.117)^{*}$ & $0.230(0.129)$ \\
\hline Neuroticism & $-0.334(0.103)^{* *}$ & $-0.231(0.138)$ \\
\hline GFP & $-0.446(0.164)^{* *}$ & $-0.197(0.202)$ \\
\hline
\end{tabular}

\subsubsection{Bias versus Gender Equality}

Table 3 contains results from the regressions of $d_{M A C S}$ against country-level gender equality. There were no significant relationships between openness or conscientiousness and bias, while there were significant - albeit marginally so with rounded unweighted model $\mathrm{p}$ values of $0.01,0.002$, and 0.01 - relationships for agreeableness, neuroticism, and the GFP. None of these survived weighting. Unlike all the rest, extraversion presented a strong negative relationship between dMACS and gender equality that was both significant and robust to weighting choices.

\section{Discussion}

Theories in the domain of personality research are typically verbally formulated and the same is true for theories about group differences in general. I believe that it is at least in no small part for this reason that their theoretical predictions are often not explicitly stated or well-known. In this study, I explained that many theories implicitly make predictions about measurement bias. Focusing on sex differences, I found that theories that make predictions about measurement bias ought to be regarded as inconsistent with the data because there is very little measurement bias in the first place. Barring this sample being markedly different from the general population in some meaningful way, virtually all theories that have been proffered as explanations for sex differences in personality are likely to be false, as they are substantial, and their substantial explanations frequently invoke phenomena that, if taken to explain sex differences, suggest sex differences are attributable to measurement bias which is, empirically, absent.

\subsection{Reemphasizing the Need for Strong Theory Testing}

The example used in this study is just one of many areas to which these same methods and sorts of inferences can be applied. All proposed instances where groups differ can and ought to be similarly examined. However, despite knowledge of techniques for assessing psychometric bias existing for more than 40 years at this point, there have been shockingly few papers about group differences that also assess if these differences can be considered real. Even worse, no papers to my knowledge have utilized this information for the explicit purpose of testing theoretical predictions. This is despite most theoretical explanations, from cultural to developmental ones, being predicated on the presence of bias.

Similar to how Heene (2013) pointed out psychologists were assuming but not testing whether their variables they purported to be measuring were quantitative, I wish 
to note that they have also not been testing whether their variables possess the same meanings between groups they aim to compare. In my time reviewing psychological work, I have often suggested to researchers that they have a duty to conduct this sort of analysis because it directly impinges on the trustworthiness of their work for two reasons. Firstly, we need to know as a baseline if group comparisons can be conceptualized as differences in the construct we intend to compare. Secondly, they need to do these analyses since their work makes predictions about the results that could force them to reinterpret the findings of their other analyses. When I have confronted them as such, only a small minority have elected to test for measurement invariance, and none have questioned their results even when faced with disconfirmation in the form of very large violations of measurement invariance. Ultimately, I can only hope that by describing the utility of these methods for discriminating between theoretical explanations, there will be some rethinking on the part of substantive researchers.

\subsection{The Gender Equality Paradox}

Because it is commonly discussed when talking about sex differences in personality and a variety of other variables, I assessed the degree to which bias and gender equality were related. Various theories make predictions about at least the directions of these effects. For example, the ideas that sex differences emerge due to different patterns of socialization or patriarchal influences (Schmitt, 2015) entail measurement bias because we cannot reasonably expect all women or all men to be affected by these things in the same ways in representative samples of the population. Unlike drugs, which may - but often do not - have homogeneous effects, it is just very rare to find a variable or constellation of variables that could plausibly act such that its effects would be hidden in this sort of analysis. These two theories should be recast as predictions about the degree to which bias will vary by country in as much as gender equality indices are genuinely related to differences in the degree to which socialization is gendered or patriarchies can influence individual development.

Since I do not subscribe to and was not attempting to test a particular explanation, the results were provided without remarks about specific theories. I found that openness and conscientiousness were unrelated to bias, and agreeableness, neuroticism, and the GFP were not robustly related to bias. Extraversion, on the other hand was negatively related to bias, such that in more gender equal countries, there was less measurement bias than in more gender-unequal countries. There are few theories presently equipped to handle these observations.

\subsection{Limitations}

The most major limitation of this study was the sampling. The sample was compromised of a very large number of individuals, but they all self-selected into taking this examination. The representativeness with respect to the general population is thus contestable and I would argue that this is probably a worse problem in less economically developed countries since the barrier required to obtain a computer, internet access, and a working knowledge of the language the test was administered in is much higher than in places like the U.S. or Australia. Moreover, to the extent this questionnaire was taken online and being online is related to social class more strongly in poorer countries, there is the risk that the data is totally irrelevant for anything to do with the Gender Equality Paradox because the sampling may systematically diverge 
from the representativeness required to render a genuine test.

The online nature of this sampling is a double-edged sword when it comes to the topic of sample size. Because the test was administered online, the potential sample was massive and, indeed, the sample size here was very large for this field. However, some number of the entries may have been from individuals retaking the test multiple times or providing answers in a given pattern in an effort to probe at the questionnaire or simply for fun. For more on concerns with the sampling here, see Johnson (2005).

The gender equality index used has questionable validity. Its relationship to individuals' experiences of gender equality is potentially dubious and may indeed be nonexistent. Moreover, because the sampling period and the gender equality index year differed, changes in the rank-ordering of countries may have impacted results that used them. Changes over time in the meaning of concepts like gender and gender equality could also have threatened the validity of my measures and results.

\section{Conclusion}

Theories entail predictions even if researchers do not state them. Group differences are considered by many to be inherently interesting and worthy of discussion and dissection. Numerous theories have been advanced to explain group differences, but the measurement-based predictions that they imply have not been explicitly tested. Using sex differences in personality as an example, I found that most theories purporting to explain why men and women differ in terms of personality will not be viable, as there is little measurement bias to account for, and in the case of a prominent topic of discussion - the relationship between gender equality and personality differences - the evidence is slanted against the utility of many commonly referenced theories. I hope these explanations and this example will aid other scholars and I believe the clear presentation of and testing for predictions about measurement can considerably improve substantial psychological theory testing.

\section{References}

Beaujean, A. A. (2014). Latent Variable Modeling Using R (1st edition). Routledge.

Benenson, J. F., Webb, C. E., \& Wrangham, R. W. (2021). Self-Protection as an Adaptive Female Strategy. Behavioral and Brain Sciences, 1-86. https://doi.org/10.1017/S0140525X21002417

Bolle, M. D., Fruyt, F. D., McCrae, R. R., Löckenhoff, C. E., Costa, P. T., Jr, AguilarVafaie, M. E., Ahn, C., Ahn, H., Alcalay, L., Allik, J., Avdeyeva, T. V., Bratko, D., Brunner-Sciarra, M., Cain, T. R., Chan, W., Chittcharat, N., Crawford, J. T., Fehr, R., ... Terracciano, A. (2015). The Emergence of Sex Differences in Personality Traits in Early Adolescence: A Cross-Sectional, Cross-Cultural Study. Journal of Personality and Social Psychology, 108(1), 171. https://doi.org/10.1037/a0038497

Booth, T., \& Irwing, P. (2011). Sex differences in the 16PF5, test of measurement invariance and mean differences in the US standardisation sample. Personality and Individual Differences, 50(5), 553-558. https://doi.org/10.1016/j.paid.2010.11.026 
Breda, T., Jouini, E., Napp, C., \& Thebault, G. (2020). Gender stereotypes can explain the gender-equality paradox. Proceedings of the National Academy of Sciences, 117(49), 31063-31069. https://doi.org/10.1073/pnas.2008704117

Costa, P. T., Terracciano, A., \& McCrae, R. R. (2001). Gender differences in personality traits across cultures: Robust and surprising findings. Journal of Personality and Social Psychology, 81(2), 322-331. https://doi.org/10.1037/0022-3514.81.2.322

Cribari-Neto, F., Souza, T. C., \& Vasconcellos, K. L. P. (2007). Inference Under Heteroskedasticity and Leveraged Data. Communications in Statistics - Theory and Methods, 36(10), 1877-1888. https://doi.org/10.1080/03610920601126589

Cuevas, Á., Cuevas, R., Desmet, K., \& Ortuño-Ortín, I. (2021). The Gender Gap in Preferences: Evidence from 45,397 Facebook Interests (Working Paper No. 29451; Working Paper Series). National Bureau of Economic Research. https://doi.org/10.3386/w29451

Del Giudice, M. (2013). Multivariate Misgivings: Is D a Valid Measure of Group and Sex Differences? Evolutionary Psychology, 11(5), 147470491301100500. https://doi.org/10.1177/147470491301100511

Del Giudice, M., Booth, T., \& Irwing, P. (2012). The Distance Between Mars and Venus: Measuring Global Sex Differences in Personality. PLoS ONE, 7(1). https://doi.org/10.1371/journal.pone.0029265

Dong, Y., \& Dumas, D. (2020). Are personality measures valid for different populations? A systematic review of measurement invariance across cultures, gender, and age. Personality and Individual Differences, 160, 109956. https://doi.org/10.1016/j.paid.2020.109956

Fokkema, M., Smits, N., Kelderman, H., \& Cuijpers, P. (2013). Response shifts in mental health interventions: An illustration of longitudinal measurement invariance. Psychological Assessment, 25(2), 520-531. https://doi.org/10.1037/a0031669

Fors Connolly, F., Goossen, M., \& Hjerm, M. (2020). Does Gender Equality Cause Gender Differences in Values? Reassessing the Gender-Equality-Personality Paradox. Sex Roles, 83(1), 101-113. https://doi.org/10.1007/s11199-019-01097-x

Giolla, E. M., \& Kajonius, P. J. (2018). Sex differences in personality are larger in gender equal countries: Replicating and extending a surprising finding. International Journal of Psychology, 54(6), 705-711. https://doi.org/10.1002/ijop.12529

Goldberg, L. R., Johnson, J. A., Eber, H. W., Hogan, R., Ashton, M. C., Cloninger, C. R., \& Gough, H. G. (2006). The international personality item pool and the future of public-domain personality measures. Journal of Research in Personality, 40(1), 84-96. https://doi.org/10.1016/j.jrp.2005.08.007

Guimond, S., Branscombe, N. R., Brunot, S., Buunk, A. P., Chatard, A., Désert, M., Garcia, D. M., Haque, S., Martinot, D., \& Yzerbyt, V. (2007). Culture, gender, and the self: Variations and impact of social comparison processes. Journal of Personality and Social Psychology, 92(6), 1118-1134. https://doi.org/10.1037/0022-3514.92.6.1118 
Hagquist, C., \& Andrich, D. (2017). Recent advances in analysis of differential item functioning in health research using the Rasch model. Health and Quality of Life Outcomes, 15. https://doi.org/10.1186/s12955-017-0755-0

Heene, M. (2013). Additive conjoint measurement and the resistance toward falsifiability in psychology. Frontiers in Psychology, 4, 246. https://doi.org/10.3389/fpsyg.2013.00246

Hyde, J. S. (2005). The gender similarities hypothesis. American Psychologist, 60(6), 581-592. https://doi.org/10.1037/0003-066X.60.6.581

Hyde, J. S. (2012, January 5). The Distance Between North Dakota and South Dakota. PLOS ONE Comments. https://journals.plos.org/plosone/article/comment?id=10.1371/annotation/2aa4d091db7a-4789-95ae-b47be9480338

Johnson, J. A. (2005). Ascertaining the validity of individual protocols from Webbased personality inventories. Journal of Research in Personality, 39(1), 103-129. https://doi.org/10.1016/j.jrp.2004.09.009

Johnson, J. A. (2014). Measuring thirty facets of the Five Factor Model with a 120item public domain inventory: Development of the IPIP-NEO-120. Journal of Research in Personality, 51, 78-89. https://doi.org/10.1016/j.jrp.2014.05.003

Kaiser, T. (2019). Nature and evoked culture: Sex differences in personality are uniquely correlated with ecological stress. Personality and Individual Differences, 148, 67-72. https://doi.org/10.1016/j.paid.2019.05.011

Kaiser, T., Giudice, M. D., \& Booth, T. (2020). Global sex differences in personality: Replication with an open online dataset. Journal of Personality, 88(3), 415-429. https://doi.org/10.1111/jopy.12500

Lasker, J., Haltigan, J. D., \& Richardson, G. B. (2021). Measurement Issues in Tests of the Socioecological Complexity Hypothesis. Evolutionary Psychological Science. https://doi.org/10.1007/s40806-021-00301-0

Lukaszewski, A. W., Gurven, M., von Rueden, C. R., \& Schmitt, D. P. (2017). What Explains Personality Covariation? A Test of the Socioecological Complexity Hypothesis. Social Psychological and Personality Science, 8(8), 943-952. https://doi.org/10.1177/1948550617697175

Marsh, H. W., Lüdtke, O., Muthén, B., Asparouhov, T., Morin, A. J. S., Trautwein, U., \& Nagengast, B. (2010). A new look at the big five factor structure through exploratory structural equation modeling. Psychological Assessment, 22(3), 471-491. https://doi.org/10.1037/a0019227

Millsap, R. E. (2005). Four Unresolved Problems in Studies of Factorial Invariance. In Contemporary psychometrics: A festschrift for Roderick P. McDonald (pp. 153-171). Lawrence Erlbaum Associates Publishers.

Musek, J. (2007). A general factor of personality: Evidence for the Big One in the five-factor model. Journal of Research in Personality, 41(6), 1213-1233. 
https://doi.org/10.1016/j.jrp.2007.02.003

Ngun, T. C., Ghahramani, N., Sánchez, F. J., Bocklandt, S., \& Vilain, E. (2011). The Genetics of Sex Differences in Brain and Behavior. Frontiers in Neuroendocrinology, 32(2), 227. https://doi.org/10.1016/j.yfrne.2010.10.001

Nye, C. D., Allemand, M., Gosling, S. D., Potter, J., \& Roberts, B. W. (2016). Personality Trait Differences Between Young and Middle-Aged Adults: Measurement Artifacts or Actual Trends? Journal of Personality, 84(4), 473-492. https://doi.org/10.1111/jopy.12173

Nye, C. D., Bradburn, J., Olenick, J., Bialko, C., \& Drasgow, F. (2019). How Big Are My Effects? Examining the Magnitude of Effect Sizes in Studies of Measurement Equivalence. Organizational Research Methods, 22(3), 678-709. https://doi.org/10.1177/1094428118761122

Orri, M., Rouquette, A., Pingault, J.-B., Barry, C., Herba, C., Côté, S. M., \& Berthoz, S. (2018). Longitudinal and Sex Measurement Invariance of the Affective Neuroscience Personality Scales. Assessment, 25(5), 653-666. https://doi.org/10.1177/1073191116656795

Richardson, G. B., \& Boutwell, B. B. (2020). Decomposition of Mean Sex Differences in Alcohol Use Within a Genetic Factor Model. Behavior Genetics, 50(5), 320-331. https://doi.org/10.1007/s10519-020-10004-0

Richardson, S. S., Reiches, M. W., Bruch, J., Boulicault, M., Noll, N. E., \& ShattuckHeidorn, H. (2020). Is There a Gender-Equality Paradox in Science, Technology, Engineering, and Math (STEM)? Commentary on the Study by Stoet and Geary (2018). Psychological Science, 31(3), 338-341. https://doi.org/10.1177/0956797619872762

Schmitt, D. P. (2015). The Evolution of Culturally-Variable Sex Differences: Men and Women Are Not Always Different, but When They Are...It Appears Not to Result from Patriarchy or Sex Role Socialization. In T. K. Shackelford \& R. D. Hansen (Eds.), The Evolution of Sexuality (pp. 221-256). Springer International Publishing. https://doi.org/10.1007/978-3-319-09384-0_11

Schmitt, D. P., Long, A. E., McPhearson, A., O'Brien, K., Remmert, B., \& Shah, S. H. (2017). Personality and gender differences in global perspective. International Journal of Psychology, 52(S1), 45-56. https://doi.org/10.1002/ijop.12265

Schmitt, D. P., Realo, A., Voracek, M., \& Allik, J. (2009). "Why can't a man be more like a woman? Sex differences in big five personality traits across 55 cultures": Correction to Schmitt et al. (2008). Journal of Personality and Social Psychology, 96(1), 118-118. https://doi.org/10.1037/a0014651

Schwab, K., Samans, R., Zahidi, S., Bekhouche, Y., Ugarte, P. P., Ratcheva, V., Hausmann, R., \& Tyson, L. D. (2015). The Global Gender Gap Report 2015 (No. 10; The Global Gender Gap Report, p. 387). World Economic Forum. https://www3.weforum.org/docs/GGGR2015/cover.pdf

Stam, D., Huang, Y.-A., \& Van den Stock, J. (2019). Non-overlapping and Inverse 
Associations Between the Sexes in Structural Brain-Trait Associations. Frontiers in Psychology, 10, 904. https://doi.org/10.3389/fpsyg.2019.00904

Stoet, G., Bailey, D. H., Moore, A. M., \& Geary, D. C. (2016). Countries with Higher Levels of Gender Equality Show Larger National Sex Differences in Mathematics Anxiety and Relatively Lower Parental Mathematics Valuation for Girls. PLOS ONE, 11(4), e0153857. https://doi.org/10.1371/journal.pone.0153857

Suzuki, T., South, S. C., Samuel, D. B., Wright, A. G. C., Yalch, M. M., Hopwood, C. J., \& Thomas, K. M. (2019). Measurement Invariance of the DSM-5 Section III Pathological Personality Trait Model Across Sex. Personality Disorders, 10(2), 114. https://doi.org/10.1037/per0000291

Udry, J. R., \& Talbert, L. M. (1988). Sex hormone effects on personality at puberty. Journal of Personality and Social Psychology, 54(2), 291-295. https://doi.org/10.1037//0022-3514.54.2.291

van de Schoot, R., Lugtig, P., \& Hox, J. (2012). A checklist for testing measurement invariance. European Journal of Developmental Psychology, 9(4), 486-492. https://doi.org/10.1080/17405629.2012.686740

van Eijk, L., Zhu, D., Couvy-Duchesne, B., Strike, L. T., Lee, A. J., Hansell, N. K., Thompson, P. M., de Zubicaray, G. I., McMahon, K. L., Wright, M. J., \& Zietsch, B. P. (2021). Are Sex Differences in Human Brain Structure Associated With Sex Differences in Behavior? Psychological Science, 32(8), 1183-1197. https://doi.org/10.1177/0956797621996664

Zell, E., Krizan, Z., \& Teeter, S. R. (2015). Evaluating gender similarities and differences using metasynthesis. The American Psychologist, 70(1), 10-20. https://doi.org/10.1037/a0038208 